\title{
Techniques d'analyses d'actinides et de radioéléments d'intérêt par spectroscopie laser*
}

\author{
C. MOULIN, A. BRIAND, P. DECAMBOX, B. FLEUROT, J.L. LACOUR,
} P. MAUCHIEN, B. REMY**

(Manuscrit reçu le 20 avril 1994)

RÉSUMÉ Les techniques de spectroscopie laser sont de plus en plus employées pour l'analyse élémentaire à bas niveau et la spéciation des actinides et autres radionucléides d'intérêt. A partir d'une recherche bibliographique et des expériences développées au sein du Laboratoire de spectroscopie laser analytique, le principe, les performances, les applications ainsi que les avantages et les inconvénients de ces différentes techniques laser sont présentés. une comparaison est effectuée avec une autre technique (non-laser) analytique performante, la torche à plasma - spectrométrie de masse. Les différentes techniques laser présentées sont en spectroscopie moléculaire : la lentille thermique, la spectrofluorimétrie laser à résolution temporelle, la fluorescence induite par laser de l'iode, et en spectroscopie atomique : la spectroscopie optogalvanique, la fluorescence induite par laser en four de graphite, la fluorescence induite par laser dans une torche à plasma, la spectroscopie d'ionisation résonnante colinéaire et l'ionisation laser couplée à la spectrométrie de masse. Les principaux avantages des techniques de spectroscopie laser sont l'absence de préparation d'échantillon, la rapidité, la sélectivité, la sensibilité ainsi que la possibilité de pouvoir effectuer des analyses à distance via fibre optique.

ABSTRACT Laser spectroscopy is more and more used for elemental analysis at low level as well as speciation of actinides and radionuclides of interest. Based on a literature review and experiments developed by the Analytical laser spectroscopy laboratory, the principle, performances, applications as well as the advantages and drawbacks of these different technique are presented. A comparison with inductively coupled plasma-mass spectrometry (ICP-MS) is presented. These different laser based spectroscopic techniques are in molecular spectroscopy : thermal lensing, time-resolved laser-induced fluorescence, fluorescence of iodine and in atomic spectroscopy : optogalvanic laser induced fluorescence in a graphite furnace, laser induced fluorescence in an ICP, collinear resonance ionization and resonance ionization mass spectrometry (RIMS). The main advantages of laser spectroscopy are the lack of chemical preparation, rapidity, selectivity, sensitivity as well as the possibility to perform remote measurements via fiber optics.

\footnotetext{
* Ce document s'inscrit dans le cadre d'une étude effectuée avec le soutien de l'Institut de protection et de sûreté nucléaire (IPSN).

** Commissariat à l'énergie atomique (CEA), DCC/DPE/SPEA/SPS/Laboratoire de spectroscopie laser analytique CE-Saclay, 91191 Gif-sur-Yvette Cedex.
} 


\section{Introduction}

Ce document fait la synthèse bibliographique des différentes techniques de spectroscopie laser utilisées pour l'analyse élémentaire et la spéciation des actinides $(\mathrm{U}, \mathrm{Np}, \mathrm{Pu}, \mathrm{Am}, \mathrm{Cm})$ et autres radioéléments d'intérêt $\left(\mathrm{Tc}, \mathrm{Sr}, \mathrm{I}_{2}\right)$ à partir d'une recherche bibliographique effectuée sur les quinze dernières années et des différentes expériences du Laboratoire de spectroscopie laser analytique (LSLA). Certaines des techniques évoquées n'ont pas encore été directement appliquées à l'analyse des actinides mais elles possèdent des potentialités intéressantes qui justifient donc leur présence dans ce document. Il est possible de scinder les techniques laser en deux catégories de spectroscopie : i) moléculaire (thermal lensing, phtoacoustique et spectrofluorimétrie laser à résolution temporelle) où il sera dans certains cas possible d'effectuer la détermination des degrés d'oxydation (spéciation) et ii) atomique (fluorescence induite par laser en torche à plasma ou four de graphite, spectroscopie d'ionisation résonante colinéaire et spectrométrie de masse - ionisation résonante) où seule l'analyse élémentaire pourra être effectuée. Le document se compose d'un descriptif de chaque technique avec son schéma de principe, les performances, les applications, les avantages et les inconvénients ainsi qu'une comparaison (voir tableau comparatif) avec une autre technique analytique (non laser) performante : l'ICP-MS (couplage torche à plasma - spectrométrie de masse).

La figure 1 présente les différents processus intervenant dans les techniques de spectroscopie laser présentées dans ce document. Dans un premier temps, il y a excitation par un laser accordé sur une transition électronique de l'élément d'intérêt, différents phénomènes peuvent être observés [32-33] :

- désexcitation non radiative : phénomènes thermique (thermal lensing) et acoustique (photoacoustique);

- désexcitation radiative : phénomènes de fluorescence moléculaire (SLRT, fluorescence de $\mathrm{I}_{2}$ ) ou atomique (fluorescence en four ou en torche à plasma);

- ionisation collisionnelle : formation d'ions par collision entre les atomes excités et les espèces moléculaires réactives dans une flamme (spectroscopie optogalvanique);

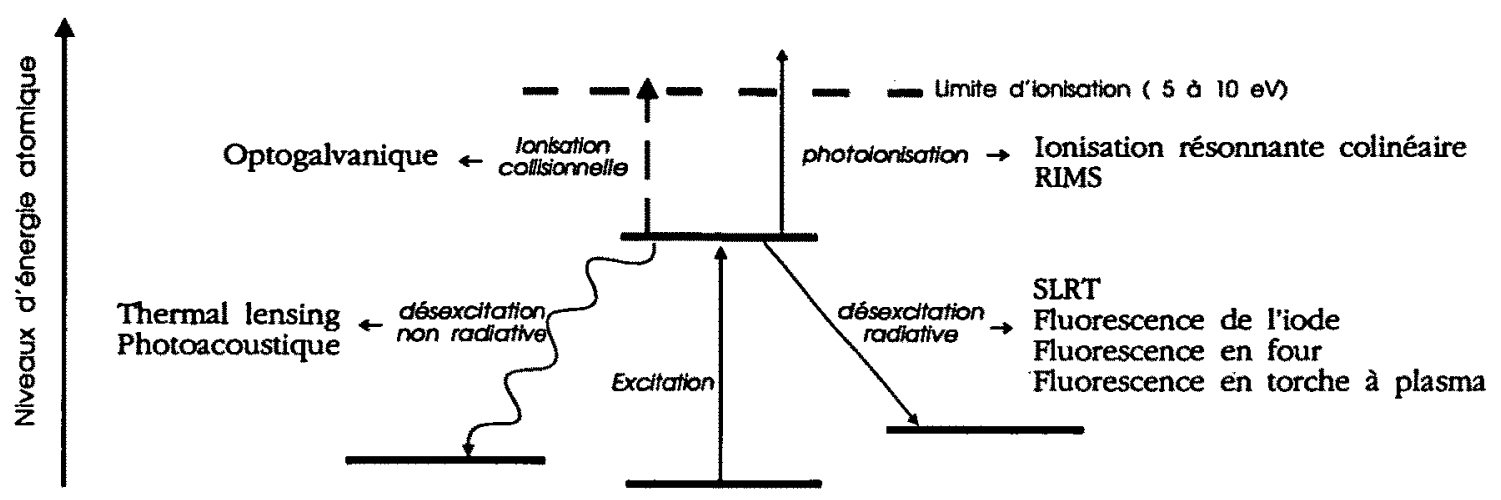

Fig. 1 - Principaux mécanismes entrant en jeu en spectroscopie laser. Main mechanisms in laser spectroscopy. 
- photoionisation : utilisation d'un ou plusieurs lasers pour formation sélective d'ions (ionisation résonnante colinéaire et RIMS).

Les sources laser utilisées pour ces différentes techniques de spectroscopie laser analytique possèdent les caractéristiques suivantes :

- Lasers pulsés : durée d'impulsion de la nano $\left(10^{-9}\right)$ à quelques dizaines de nanosecondes, énergie de quelques micro $\left(10^{-6}\right)$ à quelques millijoules $\left(10^{-3}\right)$, fréquence de quelques dizaines à quelques centaines de hertz.

- Lasers continus : énergie de quelques milliwatts à quelques watts.

\section{2. "Thermal lensing" (TL) : analyse et détermination des degrés d'oxydation des actinides (U, Pu, Am) à bas niveau $\left(\mathbf{1 0}^{-6}-\mathbf{1 0}^{-7} \mathbf{M}\right)[3,6,10,15,19,28,30,44,49]$}

\subsection{Bref historique et principe}

L'effet "thermal lensing" (la terminologie anglaise est couramment utilisée plutôt que la traduction française "lentille thermique") a été en premier reporté par Gordon en 1964 et suscite beaucoup d'intérêt comme nouvelle technique d'analyse sensible permettant la mesure de faibles absorptions d'éléments peu ou pas fluorescents en solution. Ces techniques permettent d'atteindre une limite de détection 100 à 1000 fois plus basse que la spectrophotométrie classique.

Le principe du TL est basé sur l'interaction entre un faisceau laser gaussien et un milieu contenant l'élément absorbant à doser. L'absorption à une longueur d'onde donnée et la restitution non radiative de l'énergie incidente par le milieu absorbant entraînent une élévation de température du milieu de mesure qui donne lieu à un gradient d'indice de réfraction généralement négatif formant ainsi une lentille thermique divergente. La mesure de la modification de cette divergence à l'aide d'un faisceau de sonde permet d'obtenir le signal de TL. L'étude de l'amplitude de ce signal en fonction de la longueur d'onde d'absorption permet d'obtenir les paramètres du milieu qui sont en relation avec la concentration et la nature de l'élément absorbant.

\subsection{Montage expérimental}

La figure 2 représente le montage de TL à double faisceau développé au LSLA : un laser à argon pompe deux lasers à colorant (permettant de couvrir une plage spectrale plus importante). Le faisceau colorant chopé (par un secteur tournant) est dirigé dans la cuve de mesure. L'absorption du faisceau laser par l'analyte (directement proportionnelle à la concentration) provoque l'élévation de température qui induit la formation d'une lentille thermique. Le faisceau colorant est ensuite arrêté par un filtre adapté. La lentille est analysée par un faisceau sonde hélium-néon et la détection s'effectue en champ lointain par une photodiode placée derrière un trou calibré ; le signal de la photodiode est envoyé dans un amplificateur synchronisé sur le secteur tournant. 


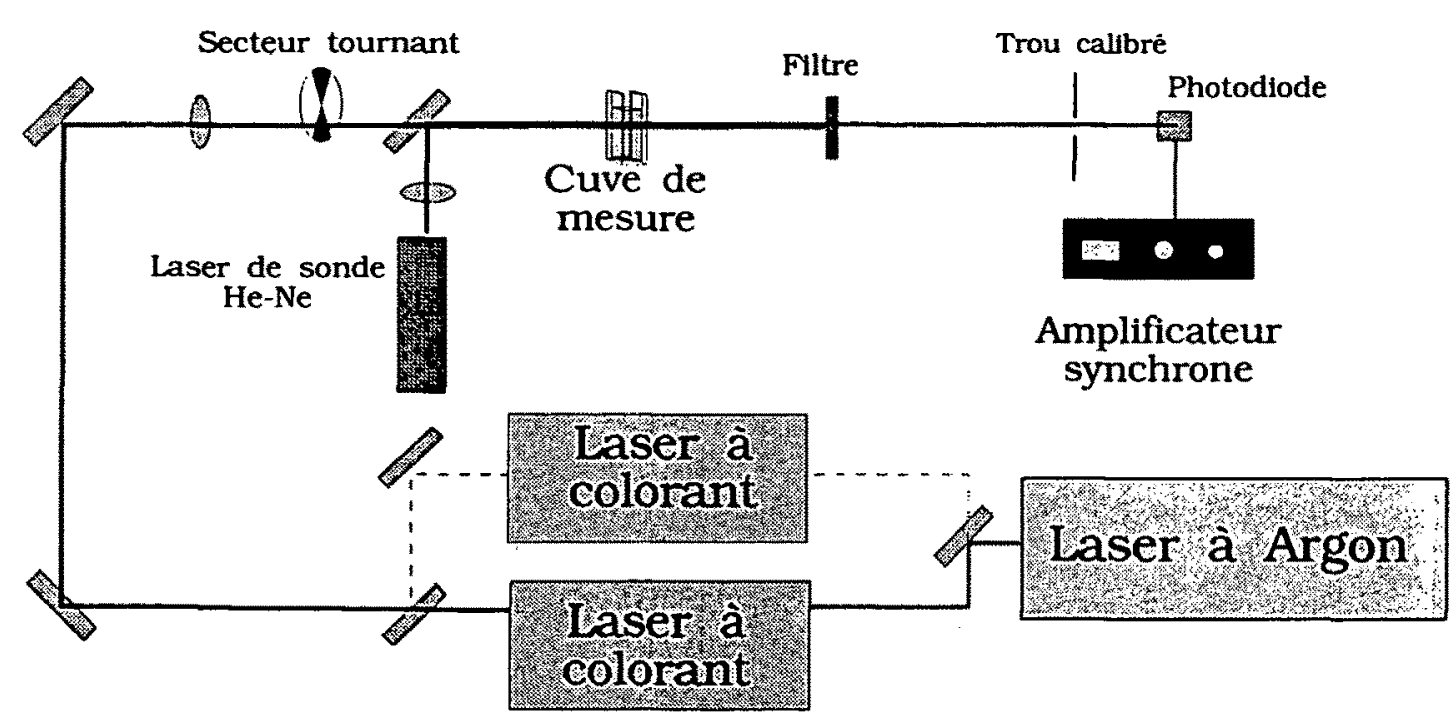

Fig. 2 - Montage de lentille thermique double faisceau.

Double beam thermal lensing set-up.

Remarque : une autre technique voisine du thermal lensing est la photoacoustique. En effet, l'absorption du rayonnement laser se traduit aussi par une onde de choc. La mesure de cette onde acoustique à l'aide d'un transducteur piézo-électrique permet d'obtenir le signal de photoacoustique. Les performances de la photoacoustique sont similaires à celles du thermal lensing.

\subsection{Performances}

Le tableau I représente les performances obtenues pour la détermination des actinides en solution par thermal lensing et photoacoustique. Dans les deux techniques, le processus mis en jeu est un transfert non radiatif (effet thermique ou onde de choc) et les limites de détection sont notamment beaucoup moins bonnes que pour des techniques optiques faisant intervenir un transfert radiatif (fluorescence par exemple). Cependant, tous les éléments absorbants peuvent être étudiés et les limites de détection sont 100 fois meilleures que celles obtenues en spectrophotométrie.

\subsection{Applications}

Le thermal lensing (TL) et la photoacoustique induite par laser sont destinés à la spéciation (caractérisation des degrés d'oxydation) et au dosage d'actinides à bas niveau.

Le montage précédent a permis de doser les lanthanides et les actinides $(\mathrm{Pu})$ à bas niveau de concentration $\left(10^{-7} \mathrm{M}<[\mathrm{Pu}]<10^{-5} \mathrm{M}\right)$ (partie grisée du tableau I). Les actinides étudiés jusqu'à présent par cette technique sont l'uranium (Ispra, Munich, Argonne, Los Alamos), l'américium, le neptunium (Munich, Harwell), le plutonium (Fontenay-aux-Roses, Munich) pour leur spé- 
TABLEAU I

Limite de détection en TL et photoacoustique pour les actinides en solution Detection limits for actinides in solution by TL and photoacoustic

\begin{tabular}{|lrr|rrr|}
\hline Espèce & $\lambda(\mathbf{n m})$ & $\mathbf{L d D}(\mathbf{M})$ & Espece & $\lambda(\mathbf{n m})$ & $\mathbf{L d D}(\mathbf{M})$ \\
\hline $\mathrm{U}(\mathrm{IV})$ & 650 & $5 \times 10^{-7}$ & $\mathrm{Pu}(\mathrm{V})$ & 569 & $4 \times 10^{-7}$ \\
$\mathrm{U}(\mathrm{VI})$ & 415 & $5 \times 10^{-7}$ & $\mathrm{Pu}(\mathrm{VI})$ & 830 & $5 \times 10^{-7}$ \\
$\mathrm{~Np}(\mathrm{III})$ & 600 & $10^{-6}$ & $\mathrm{Am}(\mathrm{III})$ & 812 & $5 \times 10^{-7}$ \\
$\mathrm{~Np}(\mathrm{IV})$ & 723 & $10^{-6}$ & $\mathrm{Am}(\mathrm{IV})$ & 650 & $5 \times 10^{-6}$ \\
$\mathrm{Pu}(\mathrm{III})$ & 610 & $2 \times 10^{-7}$ & $\mathrm{Am}(\mathrm{VI})$ & 660 & $5 \times 10^{-7}$ \\
$\mathrm{Pu}(\mathrm{IV})$ & 654 & $2 \times 10^{-7}$ & & & \\
\hline
\end{tabular}

ciation dans les eaux souterraines. Les études portent aussi sur la compréhension des équilibres chimiques des actinides à bas niveaux de concentration et sur les interactions avec les matières organiques (substances humiques) ainsi que sur l'utilisation de cette technique pour la détermination de U(VI) dans le procédé PUREX.

Les développements actuels de ces techniques, notamment aux Etats-Unis et au Japon, portent sur l'utilisation de fibres optiques pour l'analyse à distance.

- Avantages : technique de 100 à 1000 fois plus sensible que la spectrophotométrie. Spéciation de l'élément en solution.

- Inconvénients : sensibilité limitée dans le rouge et l'infra-rouge due à l'absorption de l'eau (bandes $\mathrm{OH}$ ). Montage de laboratoire uniquement (réglage optique particulièrement pointu).

\section{Spectrofluorimétrie laser à résolution temporelle : analyse des actinides et lanthanides fluorescents en solution à très bas niveau $[5,22-23,25,36,39,41-43,45-47,64]$}

\subsection{Bref historique et principe}

La fluorescence verte des sels d'uranium a été reportée pour la première fois par Becquerel en 1871. Pendant de nombreuses années, la fluorimétrie classique (avec une lampe) a été utilisée comme méthode de dosage de l'uranium. Au début des années 80, l'utilisation de laser pulsé a été reportée permettant ainsi la résolution temporelle du signal de fluorescence et le développement de nouveaux appareillages basés sur ce principe. La spectrofluori- 
métrie laser à résolution temporelle (SLRT) est une technique analytique ultrasensible pour la détermination de certains actinides $\left(\mathrm{UO}_{2}{ }^{2+}, \mathrm{Cm}^{3+}, \mathrm{Am}^{3+}\right)$ et lanthanides fluorescents en solution $\left(\mathrm{Eu}^{3+}, \mathrm{Tb}^{3+}, \mathrm{Sm}^{3+}, \mathrm{Dy}^{3+}, \mathrm{Ce}^{3+}, \mathrm{Gd}^{3+}\right.$, $\left.\mathrm{Tm}^{3+}\right)$.

Son principe est fondé sur une excitation par un laser pulsé (durée du pulse $10 \mathrm{~ns}$ ) suivie de la résolution temporelle (positionnement d'une porte de mesure quelques microsecondes après l'impulsion laser) du signal de fluorescence permettant l'élimination des fluorescences parasites (organique, Raman, Raleigh) à temps de vie courts (durée de vie $<100 \mathrm{~ns}$ ).

\subsection{Montage expérimental}

La figure 3 représente un montage de SLRT comprenant un laser $\left(\mathrm{N}_{2}, \mathrm{Nd}\right.$ YAG ou à colorant); le faisceau laser est focalisé dans la cuve de mesure et la fluorescence est collectée par un analyseur multicanal intensifié pulsé (FLUO 2001) permettant la résolution temporelle du signal de fluorescence. Ce dispositif est actuellement en fonctionnement dans différents laboratoires de I'ANDRA (Soulaines), du CEA (Cadarache, Fontenay-aux-Roses, Marcoule, BIII, Saclay), et de la COGEMA (La Hague, Marcoule).

\subsection{Performances}

Le tableau II représente les dernières performances obtenues sur les différents actinides et lanthanides en solution par spectrofluorimétrie laser à résolution temporelle.

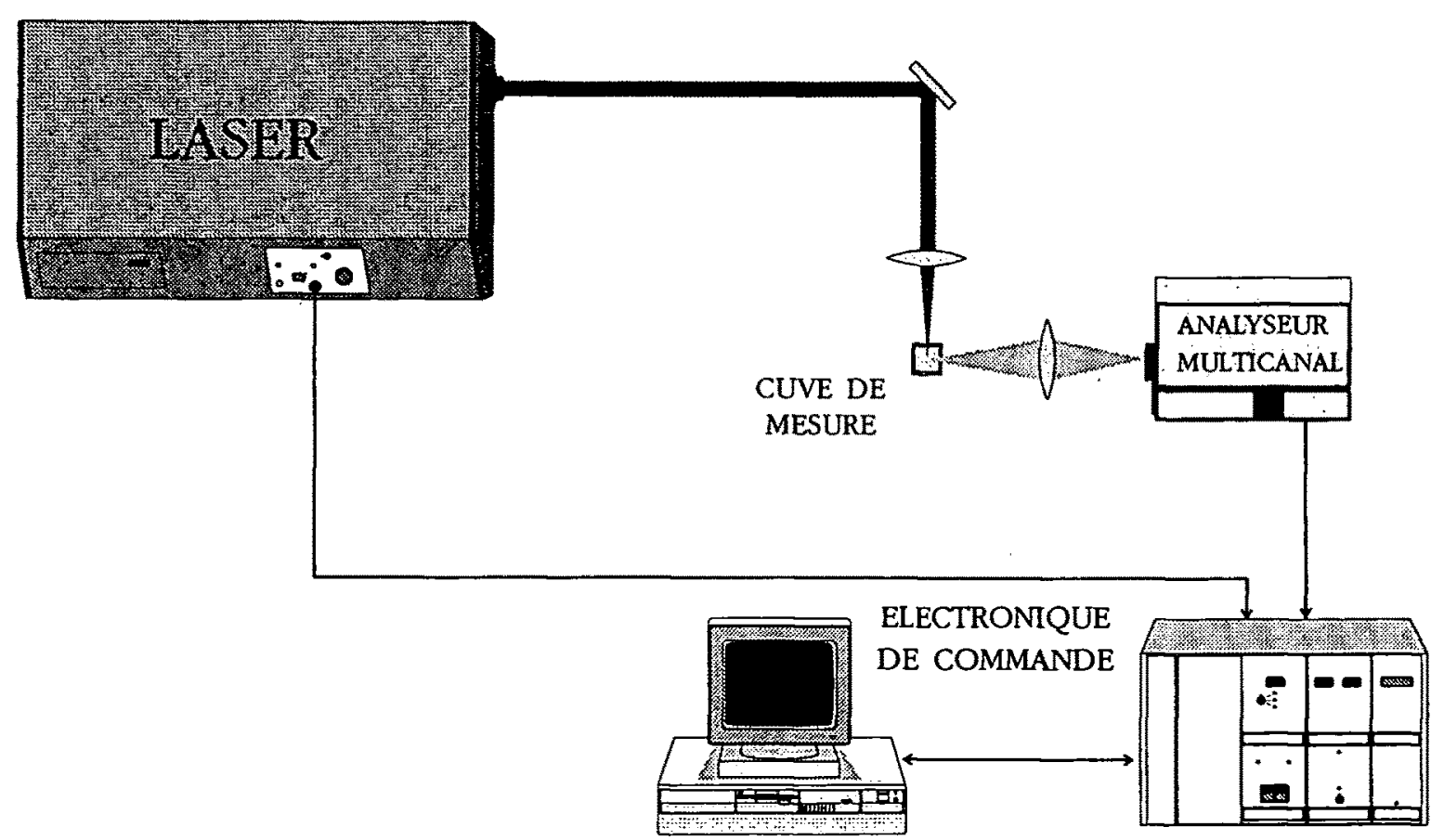

Fig. 3 - Montage de spectrofluorimétrie laser à résolution temporelle.

Time-resolved laser-induced fluorescence set-up. 
TABLEAU II

Paramètres spectroscopiques (temps de vie, longueur d'onde de fluorescence), limite de détection (LdD) dans les milieux d'analyse pour les actinides et les lanthanides par SLRT

Spectroscopic parameters (lifetime, fluorescence wavelength, limit of detection LdD) in complexing media for actinides and lanthanides by TRLIF

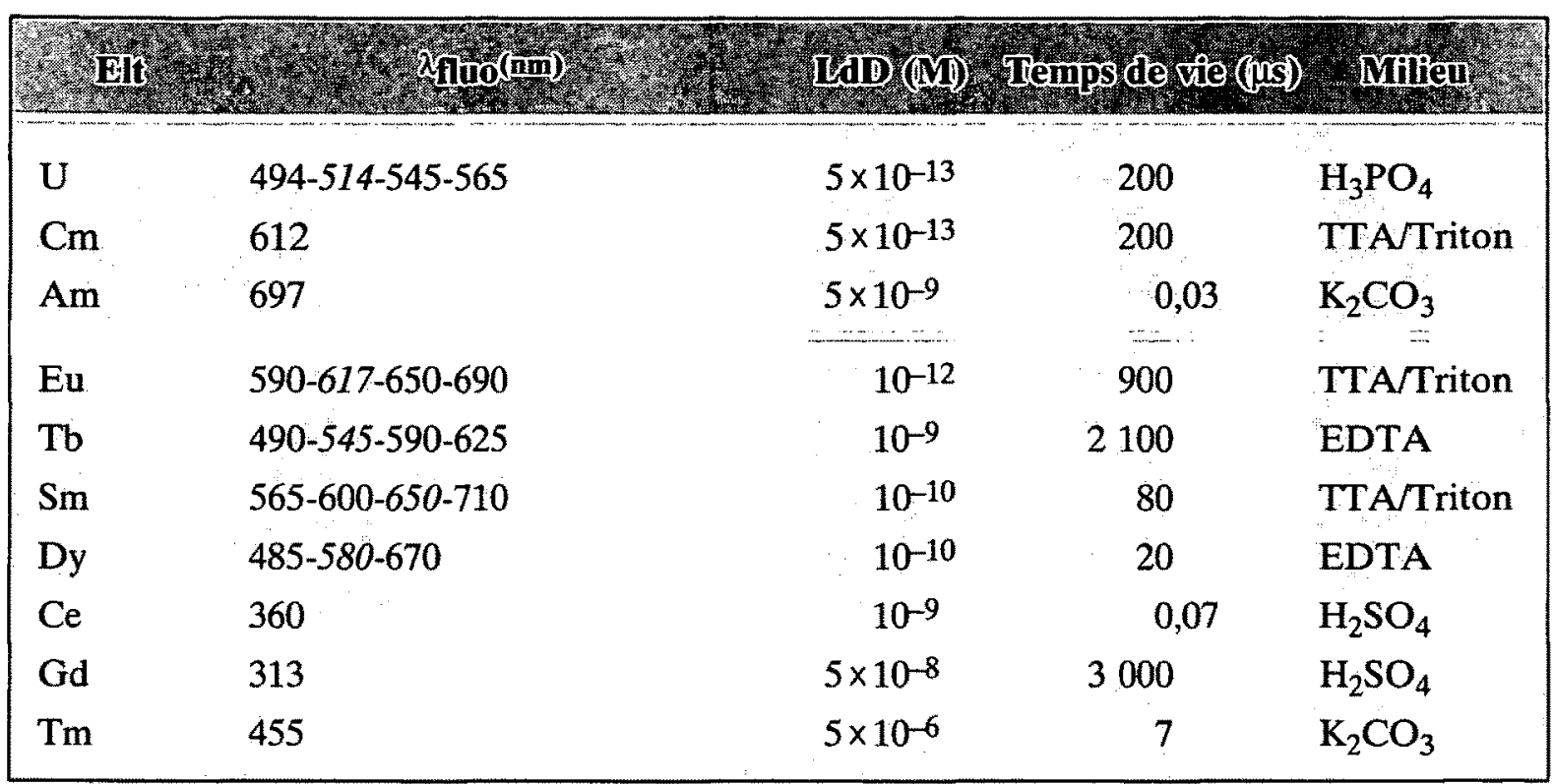

\subsection{Applications}

Cette technique développée au sein du LSLA depuis une dizaine d'années a été utilisée :

- en géologie, pour la détermination de l'uranium au niveau de la ppt $\left(5 \times 10^{-12} \mathrm{M}\right)$ dans des eaux souterraines; cette étude a permis la validation d'un modèle thermodynamique et des mesures plus précises sur la solubilité de l'uranium (VI) ;

- en chimie du procédé PUREX, pour la détermination du facteur de décontamination du TEO de UP1, pour l'analyse de l'uranium en présence de produits de fission, de plutonium, de sodium ; ...

- en contrôle médical, pour la détermination directe de l'uranium dans les urines au niveau de quelques $\mu \mathrm{g} / \mathrm{l}$ ou après minéralisation au niveau de $0,1 \mu \mathrm{g} / 1$;

- en contrôle de l'environnement, pour la détermination de l'uranium dans les terres et les eaux autour d'un centre nucléaire en vue d'une cartographie ;

- en gestion des déchets nucléaires, pour la détermination des constantes de complexation à bas niveau entre les actinides et les matières organiques (substances humiques).

- Avantages : sensibilité (limite de détection pour l'uranium et le curium : $\left.5 \times 10^{-13} \mathrm{M}\right)$, triple sélectivité ( $\lambda_{\text {excitation }}, \lambda_{\text {fluorescence }}$ et résolution temporelle), rapidité (temps d'analyse $<10 \mathrm{~min})$, dynamique $\left(>10^{6}\right.$, du ng à quelques centaines de $\mathrm{mg} / \mathrm{l})$. 
- Inconvénients : non utilisable pour les actinides non fluorescents en solution tels que $\mathrm{Pu}$ et $\mathrm{Np}$.

Les développements actuels de cette technique portent sur la spéciation (détermination des espèces chimiques) et sur l'analyse déportée via fibres optiques dans le nucléaire et dans l'environnement.

\section{Fluorescence induite par laser de l'iode moléculaire} $[9,31,40,50,52]$

\subsection{Principe}

L'iode moléculaire en phase gazeuse est excité par un laser continu chopé (par un secteur tournant) et la fluorescence ainsi induite est analysée par une détection synchrone. L'extrême finesse de l'excitation laser peut permettre, à l'aide d'un filtre intracavité (contenant un ou plusieurs isotopes complémentaires), d'exciter sélectivement l'isotope d'intérêt $\left({ }^{129} \mathrm{I}_{2}\right)$.

\subsection{Montage expérimental}

La figure 4 présente le montage développé par le LSLA pour l'analyse de l'iode moléculaire total. L'effluent gazeux circule en continu sous basse pression $(<1 \mathrm{~mm} \mathrm{Hg})$ dans une microcellule de fluorescence. Un laser à argon ionisé calé sur la raie à $514,5 \mathrm{~nm}$ excite la vapeur d'iode. L'émission de fluorescence est analysée à $90^{\circ}$ par un monochromateur couplé à un photomultiplicateur. Le signal du photomultiplicateur est traité par un amplificateur synchronisé sur le secteur tournant. L'étalonnage s'effectue par des injections d'effluents synthétiques de tension de vapeur d'iode connue. Après oxydation pyrolytique, l'iode organique est également analysé à l'état d'iode moléculaire.

\subsection{Performances}

Limites de détection $5 \times 10^{10}$ molécules $/ \mathrm{cm}^{3}\left(2 \times 10^{-5} \mathrm{~g} / \mathrm{m}^{3}\right)$. Dosage pratique jusqu'à $5 \times 10^{11}$ molécules $/ \mathrm{cm}^{3}$.

\subsection{Applications}

Dosage en ligne de l'iode et des organo-iodés. Dosage de l'iode moléculaire généré dans le dissolveur roue de l'APM à Marcoule. Possibilité d'applications aux halogènes $\left(\mathrm{Br}_{2}, \mathrm{Cl}_{2}\right)$ et à d'autres polluants colorés (dioxyde d'azote,...).

- Avantages : dosage en ligne, rapidité, possibilité d'accès à l'isotopie.

- Inconvénients : nécessité d'une dérivation. 


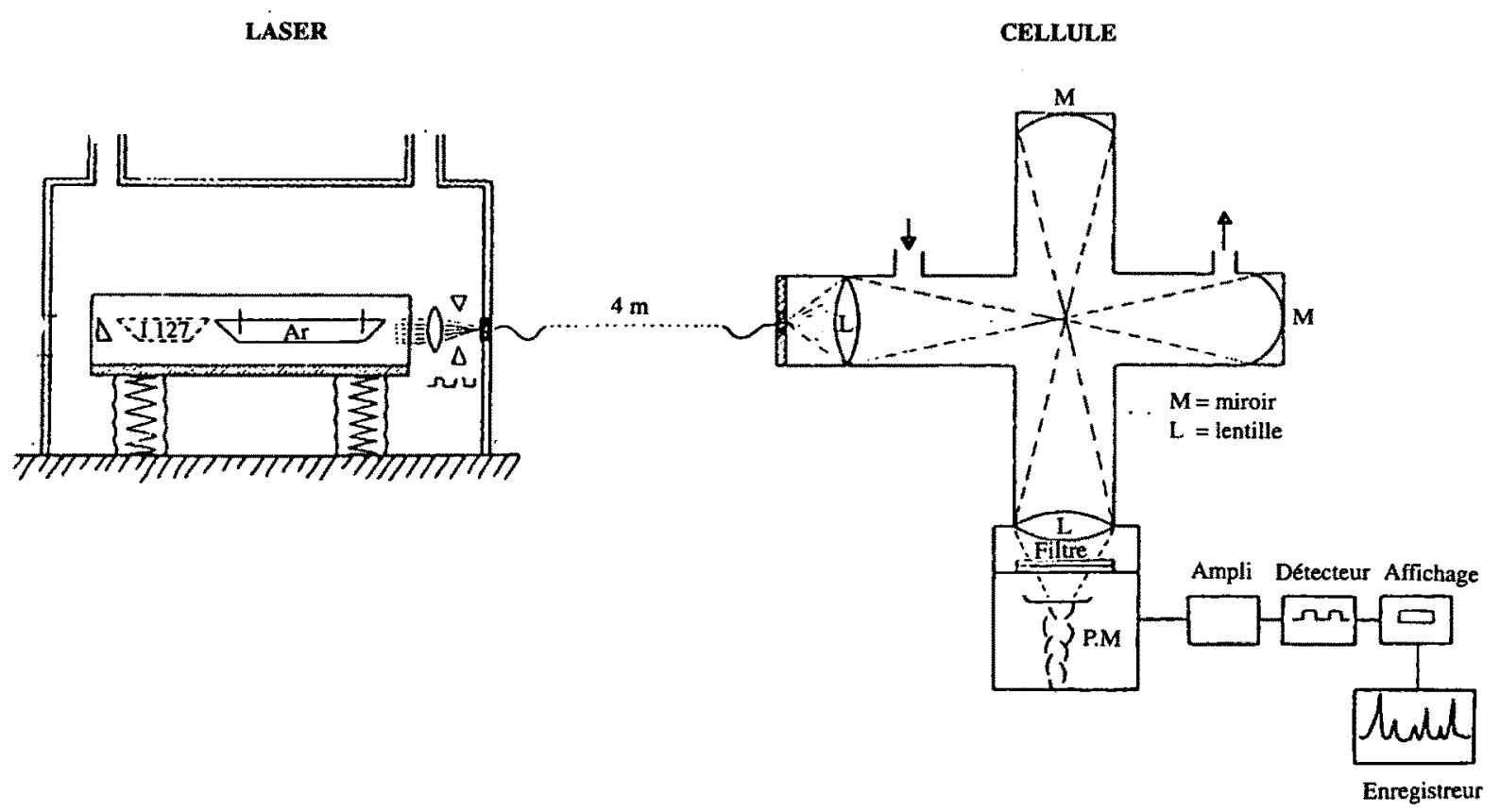

Fig. 4 - Montage de fluorescence moléculaire pour le dosage de l'iode. Molecular fluorescence set-up for iodine determination.

5. Spectroscopie optogalvanique : analyse d'ultratraces $[2,4,7,48,14]$

\subsection{Bref historique et principe}

Les potentialités de la spectroscopie optogalvanique en tant que technique d'analyse de traces sont issues des travaux du National bureau of standard (NBS) dans le milieu des années 70 . Son principe est basé sur la collection des ions crées par collision entre les molécules réactives dans une flamme air-acétylène et les atomes excités par le faisceau laser.

\subsection{Montage expérimental}

La figure 5 représente le dispositif expérimental utilisé en spectroscopie optogalvanique dans une flamme. L'échantillon est nébulisé dans un brûleur permettant l'atomisation. Un système laser pulsé (un laser excimère "pompant" un ou plusieurs lasers à colorant) est accordé sur une transition électronique appropriée de l'élément à doser. Les atomes ainsi excités sont, par collision dans la flamme, transformés en ions qui sont collectés par une électrode portée à un potentiel de -500 à $-1500 \mathrm{~V}$. Le signal électrique détecté est proportionnel aux espèces ionisés.

\subsection{Performances}

Le tableau III donne les meilleures limites de détections obtenues sur quelques éléments. 
TABLEAU III

Performances obtenues sur différents éléments en spectroscopie optogalvanique Limits of detection for several elements by optogalvanic spectroscopy

\begin{tabular}{|ccccccccc|}
\hline Elément & $\mathbf{T I}$ & $\mathbf{C a}$ & $\mathbf{G a}$ & $\mathbf{I n}$ & $\mathbf{N a}$ & $\mathbf{M g}$ & $\mathbf{L i}$ & $\mathbf{N p}$ \\
\hline LdD (ng/l) & 10 & 10 & 10 & 1 & 1 & 1 & 0,1 & 10 \\
\hline
\end{tabular}
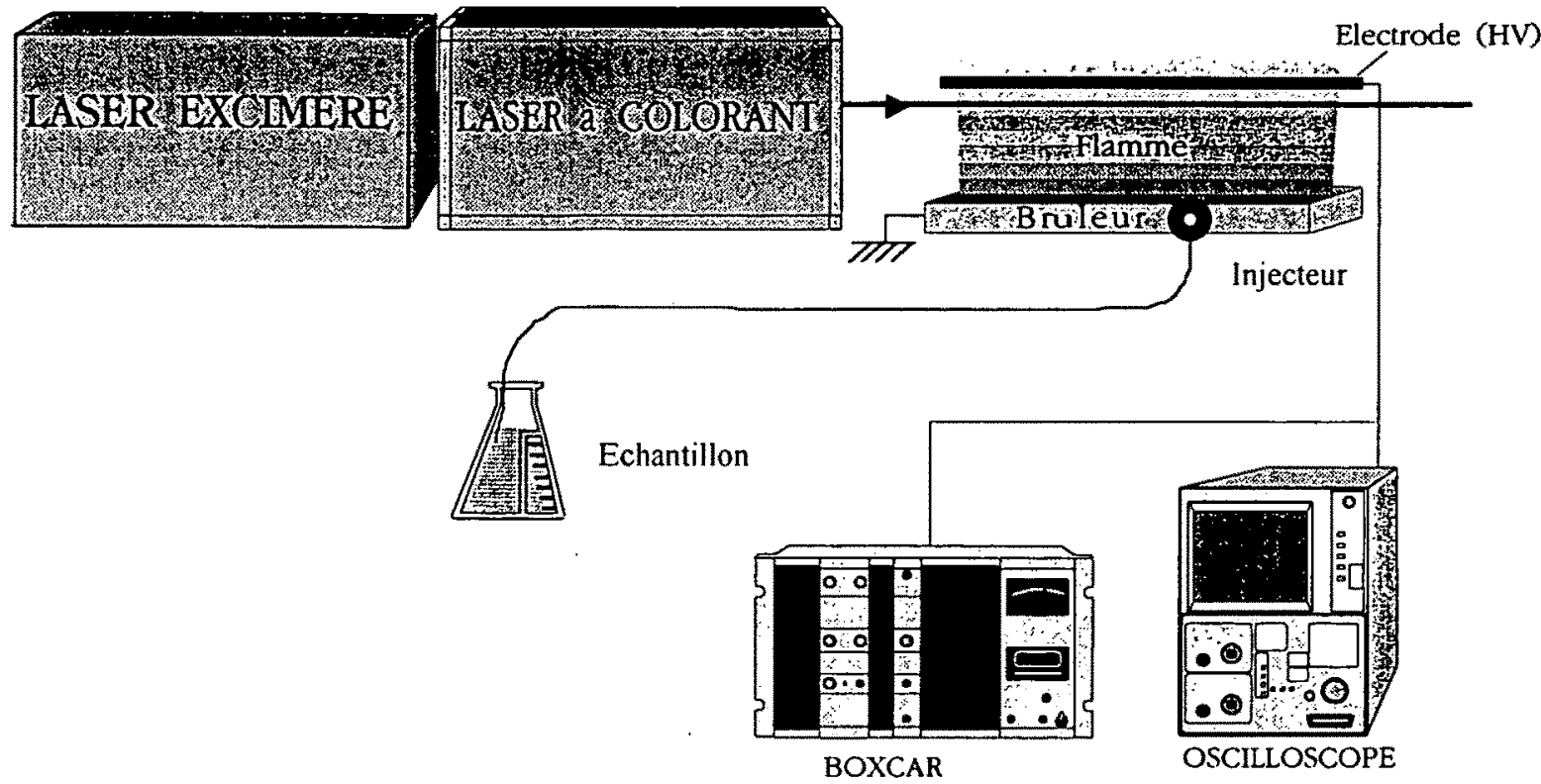

Fig. 5 - Montage de spectroscopie optogalvanique.

Optogalvanic set-up.

\subsection{Applications}

Cette technique a été utilisée pour l'analyse de nombreux éléments ( $\mathrm{Tl}, \mathrm{Co}$, $\left.\mathrm{Cs}, \mathrm{K}, \mathrm{Ni}, \mathrm{Pb}, \mathrm{I}_{2}, \ldots\right)$. En ce qui concerne les actinides, l'uranium a été étudié à des fins de spectroscopie (détermination des niveaux d'énergie), seul le neptunium a été analysé dernièrement par une équipe de Moscou avec une limite de détection pratique de $200 \mathrm{ng} / \mathrm{l}$ et une limite de détection théorique de $10 \mathrm{ng} / \mathrm{l}$.

- Avantages : applicable à la plupart des éléments, sensibilité, rapidité.

- Inconvénients : effets de matrice dus aux éléments facilement ionisables tels le sodium, effets de mémoire. Nécessité de deux couleurs ( $2 \lambda$ différentes)

\section{Fluorescence induite par laser en four de graphite : analyse d'ultratraces $[11-12,16,38,53-54]$}

\subsection{Principe}

L'atomisation thermique dans un microfour tubulaire de très faibles masses d'échantillon est suivie d'une excitation par un laser accordé sur une transition 
électronique de l'élément d'intérêt. La mesure de l'intensité de fluorescence permet de déterminer la concentration.

\subsection{Montage expérimental}

La figure 6 présente un montage de fluorescence induite par laser en four de graphite développé par le LSLA. L'échantillon est placé dans un four de graphite permettant l'atomisation. Un système laser pulsé (un laser excimère "pompant" un laser colorant) est accordé sur une transition électronique appropriée de l'élément à doser. Après excitation laser, la fluorescence de cet élément est analysée par un monochromateur couplé à un photomultiplicateur. Le signal du photomultipicateur est traité par un "boxcar" (résolution temporelle du signal de fluorescence) et un oscilloscope (visualisation du signal).

\subsection{Performances}

Cette technique a été utilisée, pour l'instant, pour doser à très bas niveau des éléments peu réfractaires. Les limites de détection (LdD) déjà obtenues sont présentées et comparées avec celles de l'absorption atomique classique dans le tableau IV.

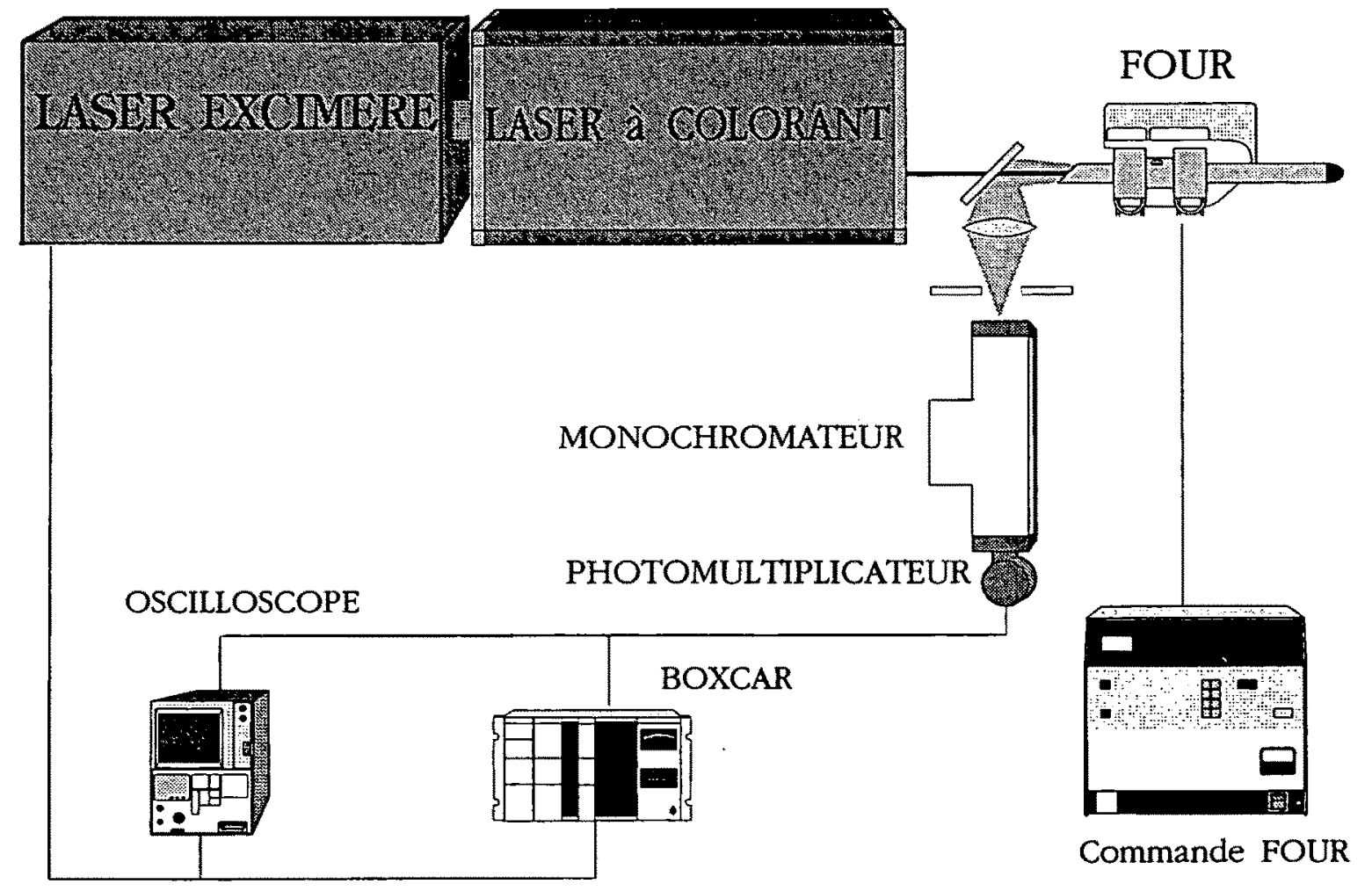

Fig. 6 - Montage de fluorescence induite par laser en four de graphite. Electrothermal atomisation-laser enhanced atomic fluorescence set-up. 
lyse des actinides dans le retraitement. Cependant, cette technique manque de sélectivité lorsque la matrice est fortement chargée (nombreuses raies d'émission non résolues). La fluorescence induite par laser dans une torche à plasma permet de lever les interférences spectrales. En effet, en accordant un laser pulsé sur une transition électronique de l'élément d'intérêt et en observant la fluorescence et non plus l'émission continue, il est possible d'analyser un élément en présence de grandes quantités d'autres éléments.

\subsection{Montage expérimental}

La figure 7 présente le montage de fluorescence induite par laser dans une torche à plasma développé par le LSLA. L'échantillon à doser est introduit dans une torche à plasma $(T>5000 \mathrm{~K})$ via un nébuliseur. Un système laser pulsé (un laser excimère "pompant" un laser à colorant) est accordé sur une transition électronique appropriée de l'élément à doser. La fluorescence de cet élément est analysée par un spectromètre haute résolution couplé à une détection pulsée.

\subsection{Performances}

Cette technique permet de doser les actinides avec les mêmes performances qu'en spectrométrie d'émission dans une torche à plasma, soit quelques ppb, mais sans interférences dans le cas des matrices chargées.

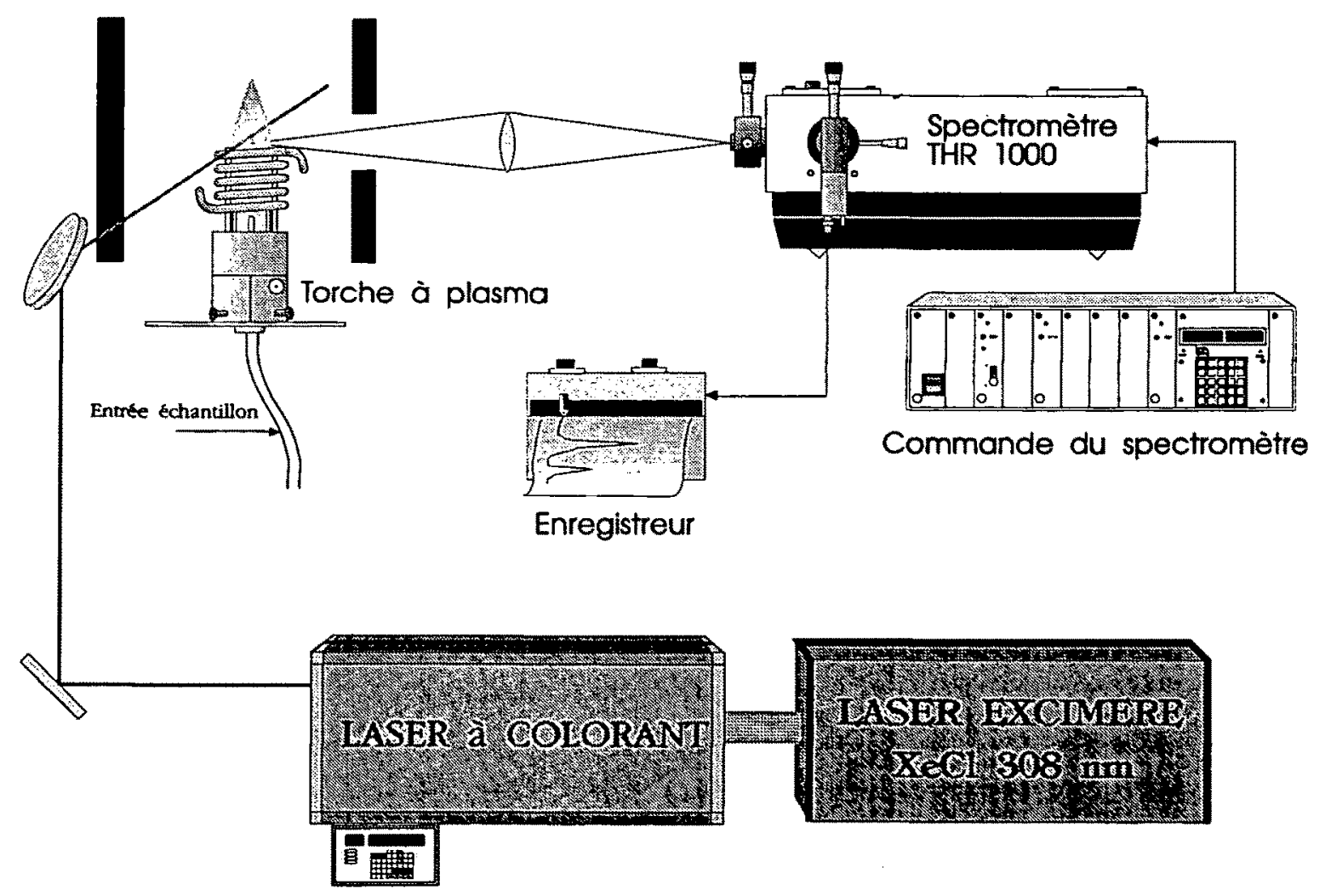

Fig. 7 - Montage de fluorescence induite par laser dans une torche à plasma. Inductively coupled plasma-laser induced fluorescence set-up. 


\subsection{Applications}

La fluorescence induite par laser dans une torche à plasma a été utilisée à La Hague dans le cas d'expériences de faisabilité pour l'analyse du Pu en présence de grandes quantité d'uranium. De plus, d'autres éléments tels $\mathrm{Np}$ et Tc pourraient être analysés en présence de fortes concentrations en uranium, une fois les bons schémas spectroscopiques trouvés. Les systèmes laser actuellement utilisés pourraient être remplacés par des diodes laser pour une plus grande souplesse.

- Avantages : sélectivité, facilité de mise en œuvre, applicable à tous les actinides.

- Inconvénients : sensibilité au niveau de la ppb, nécessité d'un schéma spectroscopique adapté.

\section{Spectroscopie d'ionisation résonante colinéaire : analyse rapide des isotopes radioactifs du strontium $\left({ }^{90} \mathrm{Sr}\right.$ et ${ }^{89} \mathrm{Sr}$ ) en présence de strontium naturel (88Sr) [59-61]}

\subsection{Principe et bref historique}

Actuellement, la seule équipe ayant mis au point une telle expérience est l'équipe du Pr Trautmann à Mayence (Allemagne). Pour atteindre l'objectif de l'analyse, il faut atteindre une sélectivité isotopique de $10^{11}$ (échantillon typique $10^{18}-10^{19}$ atomes de ${ }^{88} \mathrm{Sr}$ et $10^{8}$ atomes de ${ }^{90} \mathrm{Sr}$ dans $1000 \mathrm{~m}^{3}$ après Tchernobyl). La sélectivité dans la "resonance ionization mass spectrometry" (RIMS) est le produit de celle de l'excitation optique et celle d'un spectromètre de masse.

En effet, avec un spectromètre de masse, une sélectivité isotopique de $10^{5}-10^{6}$ est réalisable. Pour atteindre la sélectivité supplémentaire de $10^{5}-10^{6}$ avec l'excitation optique pour le strontium dont les décalages isotopiques sont très faibles, il faut recourir à une technique élaborée où les décalages en longueur d'onde entre les isotopes sont élargis artificiellement par effet Doppler (description dans la partie expérimentale). C'est une expérience complexe de très grande envergure, les allemands ont une équipe de 10 personnes depuis 4 ans sur ce sujet.

\subsection{Montage expérimental}

La figure 8 présente le montage de spectroscopie d'ionisation résonante colinéaire développé par l'équipe de Mayence. Après extraction chimique, l'échantillon est introduit dans une source d'ions où les ions formés (après atomisation et ionisation sur un filament au tantale T $2400^{\circ} \mathrm{C}$ ) sont accélérés à $50 \mathrm{keV}$. Une première suppression du ${ }^{88} \mathrm{Sr}$ naturel est obtenue par un champ magnétique $\left(60^{\circ}\right)$, après déflexion des ions de $10^{\circ}$ pour être colinéaire au faisceau laser, un échange de charge avec une vapeur de $\mathrm{Cs}\left(10^{-3} \mathrm{mbar}\right)$ pour pro- 
duire un faisceau d'atomes est effectuée. Puis, l'isotope d'intérêt est excité par des lasers continus monomodes et, après ionisation par effet de champ et déflexion par un champ électrique, les ions sont détectés par un channeltron.

\subsection{Performances}

La sélectivité isotopique obtenue est de $10^{11}$ et la limite de détection actuelle de $5 \times 10^{7}$ atomes sur ${ }^{90} \mathrm{Sr}$. Des expériences ont eu lieu courant 93 sur des échantillons réels.

\subsection{Applications}

Dosage des isotopes du strontium dans des échantillons gazeux (notamment pour le contrôle par les Allemands des rejets de la centrale nucléaire de Cattenom). Applications à d'autres éléments.

- Avantages : rapidité ( 2 jours), prélèvement limité à $1000 \mathrm{~m}^{3}$ (la méthode radiochimique demande 15 jours (équilibre séculaire de l'yttrium) et un prélèvement de $10000 \mathrm{~m}^{3}$ ).

- Inconvénients : technique lourde à mettre en œuvre.

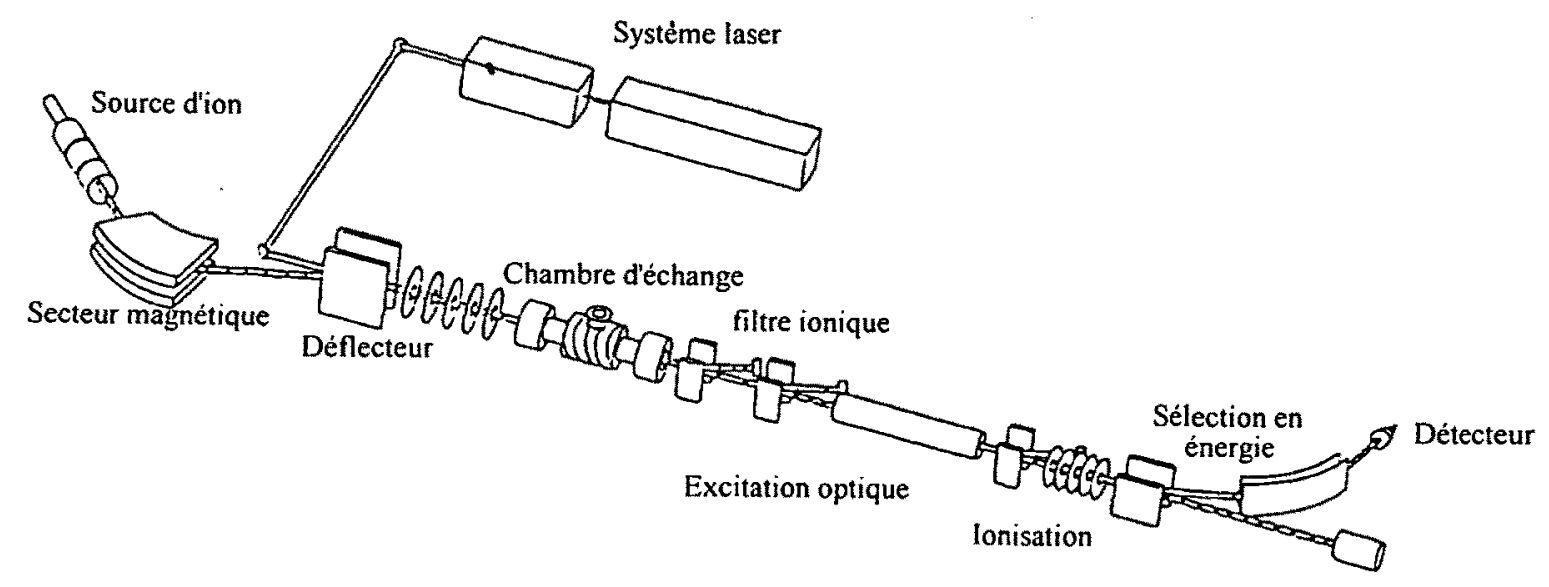

Fig. 8 - Montage de spectroscopie d'ionisation résonante colinéaire.

Colinear resonance ionization set-up.

\section{Ionisation laser - Spectrométrie de masse}

$[11,20-21,24,26-27,55-58,63]$

\subsection{Principe et bref historique}

C'est dans le début des années 80 qu'une nouvelle technique analytique, la spectrométrie de masse à ionisation résonante (la terminologie anglaise RIMS (resonance ionization mass spectroscopy) est la plus souvent employée) permettant de résoudre les problèmes d'interférences isobariques (grâce à la possibilité d'opérer une sélectivité élémentaire parfaite au niveau de l'ionisation) 
et ainsi d'éviter les séparations chimiques a vu le jour. Cette technique repose sur la spectroscopie d'ionisation résonnante :

- irradiation d'un mélange d'atomes en phase vapeur par un rayonnement laser qui permet d'exciter puis d'ioniser sélectivement les seuls atomes dont la transition électronique correspond à la longueur d'onde laser ;

- utilisation d'un système dispersif en masse (analyseur magnétique, spectromètre à temps de vol) qui permet d'obtenir la double sélectivité, élémentaire et isotopique.

\subsection{Montage expérimental}

La figure 9 représente le montage simplifié d'une installation de RIMS. Un système laser composé d'un laser de pompe (excimère, $\mathrm{Nd}$-YAG triplé ou vapeur de cuivre) couplé à un laser à colorant permet d'avoir l'accordabilité en longueur d'onde. Ce rayonnement laser accordé sur une transition électronique d'un élément d'intérêt est envoyé dans une vapeur atomique (préalablement formée par chauffage de l'échantillon sur un filament), l'élément est excité sélectivement puis ionisé et les ions ainsi formés sont envoyés dans le spectromètre de masse, puis détectés par un système intensifié.

Chambre à vide

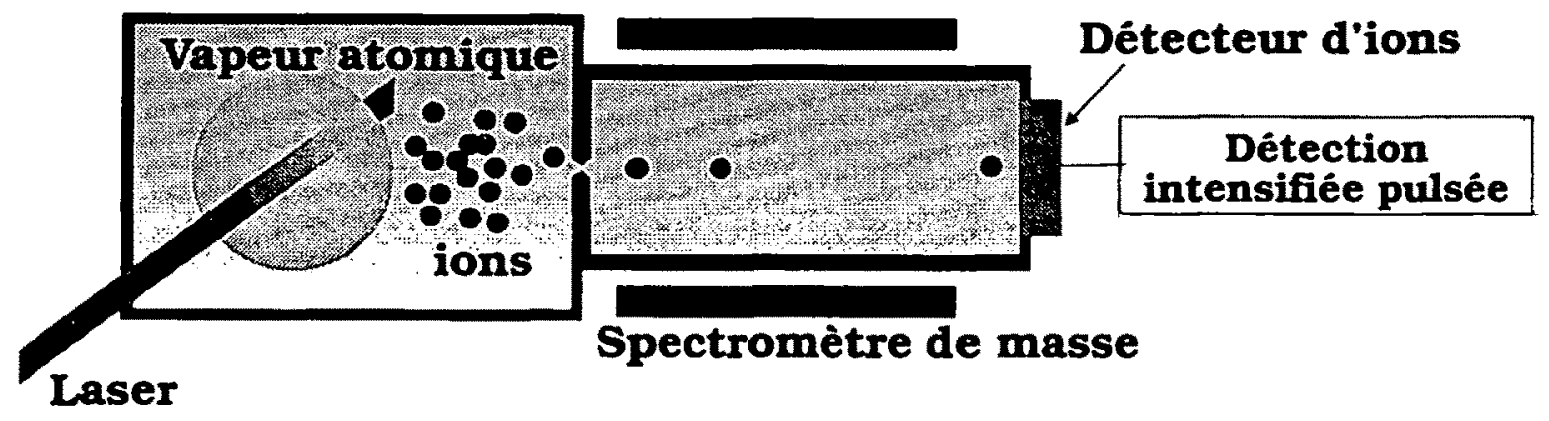

Fig. 9 - Montage simplifié de RIMS.

Schematic of RIMS set-up.

\subsection{Performances}

Concernant les actinides, les principaux travaux publiés sont de nouveau ceux de l'équipe du Pr Trautmann à Mayence; les Américains de Los Alamos travaillent aussi sur ce sujet mais publient peu. Les limites de détection obtenues sur les isotopes d'intérêt de $\mathrm{U}, \mathrm{Pu}$, Tc sont de l'ordre de $10^{6}-10^{7}$ atomes (par comparaison, en spectrométrie $\alpha$, une limite de détection de $0,4 \mathrm{mBq}$ de ${ }^{239} \mathrm{Pu}$ correspond à $4 \times 10^{8}$ atomes).

\subsection{Applications}

Détermination à très bas niveau des isotopes du Pu dans les échantillons de l'environnement. Contrôle de l'impact écologique des installations nucléaires. 
- Avantages : très grande sensibilité, bonne résolution isotopique, applicable à la plupart des radionucléides.

- Inconvénients : technique assez lourde à mettre en œuvre, personnel qualifié nécessaire.

Le tableau $\mathrm{V}$ récapitule les différentes performances des techniques de spectroscopie laser pour l'analyse de traces et les comparent avec celles de l'ICP-MS (couplage d'une torche à plasma et d'un spectromètre de masse), technique analytique très performante pour l'analyse à bas niveau [33].

TABLEAU $\mathrm{V}$

Comparaison des performances des différentes techniques

de spectroscopie laser pour l'analyse et de l'ICP-MS

Performance comparison between ICP-MS

and laser spectroscopy based techniques

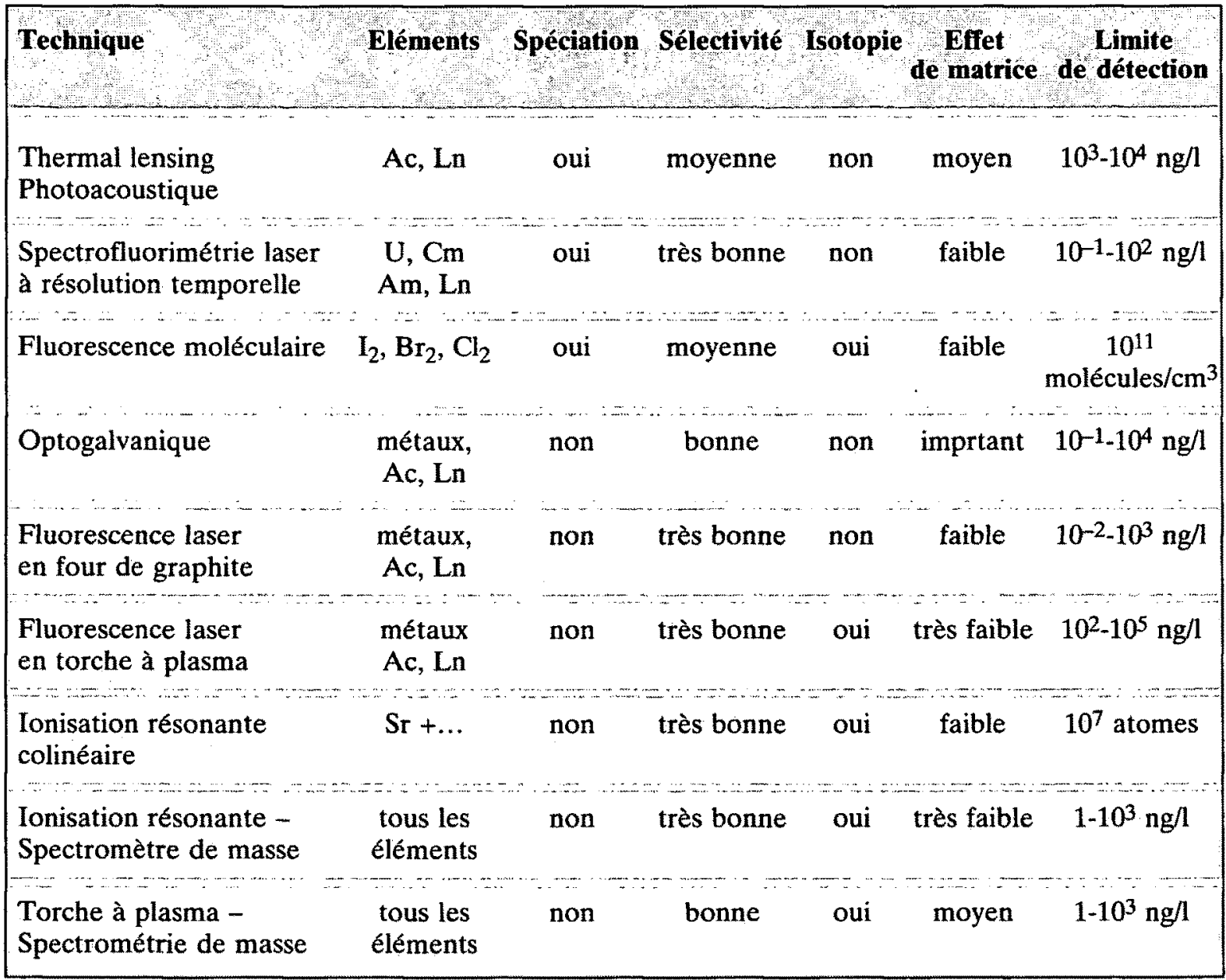

Ac actinides

Ln lanthanides

\section{Conclusion}

Les techniques de spectroscopie laser analytique présentées possèdent un certain nombre de caractéristiques bien spécifiques : 
- pas ou peu de préparation d'échantillon,

- rapidité (dosage effectué en quelques dizaines de minutes),

- sélectivité (peu ou pas d'effets de matrice),

- sensibilité (limite de détection proche de la ppt (ng/l)),

- possibilité de travailler sur des microvolumes,

- possibilité de mesure déportée ou en ligne (directement ou par fibres optiques), SLRT,

- accès aux espèces chimiques (spéciation dans le cas du TL, PAIL et néaire.

- accès à l'isotopie dans le cas du RIMS et de l'ionisation résonante coli-

Elles offrent donc potentiellement un grand intérêt pour l'analyse des actinides et des radioéléments à l'état de traces. Ces techniques souffrent, par contre, de certaines limitations qui proviennent de la complexité des expériences (connaissance de la spectroscopie des éléments d'intérêt, compréhension des mécanismes physiques et chimiques, utilisation des lasers à colorant...). Leur mise en œuvre nécessite, le plus souvent, du personnel qualifié. La seule technique développée dans le laboratoire qui connaisse un développement instrumental est la SLRT (FLUO 2001) qui utilise simplement un laser à azote ou un laser Nd-YAG triplé. D'autres techniques utilisant des lasers simples sont de plus en plus utilisées dans l'industrie (spectroscopie Raman, ablation laser, ...).

Cette synthèse non exhaustive n'aborde pas les possibilités de l'analyse directe de solides par spectroscopie laser. L'ablation laser, qui consiste à focaliser un laser de puissance à la surface de l'échantillon pour le vaporiser, peut être utilisée comme source d'atomisation de microéchantillons solides. L'absence de préparation d'échantillon et la possibilité d'opérer à pression atmosphérique constituent deux atouts majeurs de cette technique [1, 34-35, 37, 51].

La technologie laser évolue actuellement vers des systèmes plus compacts et plus simples (diode laser [17]). Ceux-ci présentent un intérêt majeur pour les actinides. Il nous semble donc important d'effectuer les études de base visant à évaluer le champ d'application de ces techniques dans les problèmes liés à l'environnement.

\section{RÉFÉRENCES}

[1] ALLMEN V. - Laser beam interactions with materials. Berlin : Springer Verlag, 1987.

[2] BAJAJ P.N. - Two colour multiphoton ionization spectroscopy of uranium from a metastable state. Appl. Phys. B., 1988, 47, 55-59.

[3] BERG J.M., MORRIS D.E., CLARK D.L., TAIT C.W., WOODRUFF H., VAN DER SLUYS W.G. - Pulsed photothermal spectroscopy applied to lanthanide and actinide speciation. In : Optical methods for ultrasensitive detection and analysis. SPIE. 1991, 1435, 331-337. 
[4] BERTHOUD T., BENCHEIDA M., MAUCHIEN P. - Spectroscopie optogalvanique dans la flamme. J. Phys. Colloq. France, 1987, 48, C7-747-751.

[5] BERTHOUD T., DECAMBOX P., MAUCHIEN P., MOULIN C. - Direct uranium trace analysis in plutonium solutions by time-resolved laser-induced fluorescence. Anal. Chem., 1988, 60, 1296-1299.

[6] BERTHOUD T., DELORME N. - Differential dual-beam thermal lensing spectrometry : determination of lanthanides. Appl. Spectrosc., 1987, 41, 15-19.

[7] BERTHOUD T., DRIN N., LIPINSK Y., CAMUS P. - Atomic analysis instrument using optogalvanic spectroscopy. J. Phys. Colloq. France, 1983, 44, C7-67-74.

[8] BERTHOUD T., MAUCHIEN P., VIAN A., LE PROVOST P. - Laser-induced fluorescence of plutonium in ICP : applications to nuclear fuel reprocessing analytical control. Appl. Spectrosc., 1987, 41, 913-914.

[9] BERTHOUD T., REMY B. - Fluorescence de l'iode stimulée par laser : application au dosage de l'iode 129 dans les effluents gazeux. Rapport technique SEA-478, 1982.

[10] BIDOGLIO G., OMENETTO N., CAVALI P., ROSSI G., TURCK G.C. - Thermal lensing spectrophotometry of uranium (VI) with pulsed laser excitation. J. Anal. At. Spectrom., 1987, 2, 579-583.

[11] BOLSHOV M.A., BOUTRON C.F., APATIN V.M., ARKHANGELSKII B.V., ERMOLOV V.V., KOLOSHNIKOV V.G., KOMPANETZ O.N., KUZNETSOV N.I., MIKHAILOV V.S., SHISHKOVSKII V.S. - Automated laser excited atomic fluorescence spectrometer for determination of trace concentrations of elements. Spectrochim. Acta B, 1989, 44, 253-258.

[12] BOLSHOV M.A., BOUTRON C.F., DUCROZ M. GÖRLACH U., KOMPANETZ O.N. RUDNIEV S.N., HUTCH B. - Direct ultratrace determination of $\mathrm{Cd}$ in Antarctic and Greenland snow and ice by laser atomic fluorescence spectrometry. Analyt. Chim. Acta., 1991, 251, 169-175.

[13] BRIAND A. - Réalisation d'un appareillage de spectrométrie de masse à ionisation résonante : application à l'étude spectroscopique et analytique de terres rares. Mémoire CNAM, Paris, 1986.

[14] CHEKALIN N.V., MARUNKOV A.G., PAVLUTSKAYA V.I., BACHIN S.V. - A rod flame system in direct laser enhanced ionization analysis of high purity substances. Spectrochim. Acta B, 1991, 46, 551-555.

[15] DOXTRADER M.M., BEITZ J.V., MARONI V.A. - Laser photoacoustic spectroscopy for trace level detection of actinides in groundwater. Mat. Res. Soc. Symp. Proc., 1987, 84, 473180.

[16] DUHAU L. - Evaluation des performances de la spectroscopie de fluorescence atomique induite par laser dans un four de graphite pour l'analyse élémentaire dans des échantillons biologiques. Rapport interne CEA-DCAEA/SEA/SEACC/LSLA, 1988.

[17] EDELSON R.J., LEE S.C., LIPERT M.C. - Application of diode lasers to actinide atom monitoring. Appl. Spectrosc., 1992, 46, 1307-1308.

[18] EDELSON M.C., VERA J.A., MURRAY G.M., WEEKS S.J. - Isotopic abundance determination by ICP high-resolution laser excited atomic and ionic fluorescence spectroscopy. Spectrochim. Acta B, 1991, 13, 1689-1700.

[19] FANG H.L., SWOFFORD R.L. - The thermal lens in absorption spectroscopy. In : Ultrasensitive laser spectroscopy. New York : Academic Press 1983, 175-231.

[20] FASSET J.D., TRAVIS J.C. - Analytical applications of resonance ionization mass spectrometry (RIMS). Top. Laser Spectrosc., 1988, 4, 1409-1422.

[21] HERMANN G., RIMKE H., AMES F., MANG M., MUHCLEK C., OTTEN E.W., REKLAU D., RIEGEL J., RUSTER W., SATTELBERGER P., SCHEERER F. - Laser spectroscopy as an analytical technique for process control in waste management, final research report. Bonn : Public ministry for research and technology, 1989. 
[22] HONG K.B., JUNG K.W., JUNG K.H. - Application of laser-induced fluorescence for determination of trace uranium, europium and samarium. Talanta, 1989, 36, 1095-1099.

[23] HUBERT S., THOUVENOT P., MOULIN C., DECAMBOX P., MAUCHIEN P. Americium trace determination in aqueous and solid matrices by TRLIF. Radiochim. Acta, $193,61,15-21$.

[24] HURST G.S., PAYNE M.G. - Principle and applications of resonance ionisation spectroscopy. Bristol : A. Hilger, 1988.

[25] ISHIBASHI K., SAKAMAKI S. - Determination of uranium at ultratrace levels by timeresolved laser fluorimetry. Anal. Chim. Acta, 1989, 219, 181-190.

[26] JOHNSON S.G., RIOS E.L., MILLER C.M., FEAREY B.L. - High efficiency resonance ionization mass spectrometric analysis by external laser cavity enhancement techniques. In : SPIE OE. LASER 91, Los Angeles, CA, January 20-25 1991, 1991, 409-414.

[27] JOHNSON S., FERAY B. - Spectroscopic study of thorium using continuous wave resonance ionization mass spectrometry with ultraviolet ionization. Spectrochim. Acta B, 1993, 48, 1065-1068.

[28] KIM J.I., BUCHAU G., BRYANT E., KLENZE R. - Complexation of americium (III) with humic acid. Radiochim. Acta, 1989, 48, 135.

[29] KIMURA T., NAKAYAMA S., TAKAHASHI S., TAKEISHI R. Speciation of uranium in aqueous solutions and in precipitates by photoacoustic spectroscopy. Radiochim. Acta, 1992, 58/59, 173-178.

[30] KLENZE R., KIM J.I., WIMMER H. - Speciation of actinides ions by pulsed laser spectroscopy. Radiochim. Acta, 1991, 52/53, 97.

[31] KUNO Y. - On-line determination of $I_{2}$ in nuclear fuel reprocessing off-gas streams by a combination of laser induced fluorimetry and laser photoacoustic spectroscopy. Anal. Chim. Acta, 1992, 270, 181-185.

[32] MAUCHIEN P. - Spectrofluorométrie moléculaire et spectrométrie de fluorescence atomique. In : Les techniques de l'ingénieur, Paris, 1990, 2835-1 à 12.

[33] MAUCHIEN P., SJOSTRÖM S. - Laser atomic spectroscopic techniques - the analytical performance for trace element analysis of solid and liquid samples. Spectrochim. Acta Rev., $1993,15,153-180$.

[34] MAUCHIEN M., AUTIN M., BRIAND A., MERMET J.M. - Characterisation by emission spectrometry of a laser produced plasma from a copper target in air at atmospheric pressure. Spectrochim. Acta B, 1993, 48, 851-856.

[35] MAUCHIEN P., ANDRE N., LACOUR J.L., GEERSTEN C. - UV laser ablation optical emission spectrometry on aluminium alloys in air at atmospheric pressure. Spectrochim. Acta B, 1994 (à paraître).

[36] MAUCHIEN P., DECAMBOX P., BERTHOUD T., KIRSCH B., MOULIN C. - Direct determination of traces of lanthanide ions in aqueous solutions by TRLIF. Anal. Chim. Acta, $1989,220,235-241$.

[37] MAUCHIEN P., GEERSTEN C., BRIAND A., CHARTIER F., LACOUR J.L., SJOSTRÖM S., MERMET J.M. - Comparison between IR and UV laser ablation at atmospheric pressure : implications for solid sampling ICP. J. Anal. Atom. Spectrom., 1994, 9, 17-22.

[38] MAUCHIEN P., REMY B., VERHAEGUE I. - Real sample analysis by ETA-LEAFS with background correction : application to gold determination in river water. Appl. Spectrosc., $1990,44,1633-1638$.

[39] MAUCHIEN P. - Dosage de l'uranium par spectrofluorimétrie à source d'excitation laser. Rapport CEA-R-5300, 1985.

[40] McDONALD J.R. - Radioidine detector based on laser induced fluorescence. Brevet USA $n^{\circ} 4188120,1978$. 
[41] MOULIN C., BEAUCAIRE C., DECAMBOX P., MAUCHIEN P. - Determination of uranium in solution at the $\mathrm{ng} / \mathrm{l}$ level by TRLIF : application to geological survey. Anal. Chim. Acta, 1990, 238, 291-296.

[42] MOULIN C., DECAMBOX P., MAUCHIEN P. - Determination of curium at $0.1 \mathrm{ng} / \mathrm{l}$ in a micellar medium by TRLIF. Anal. Chim. Acta, 1991, 254, 145-151.

[43] MOULIN C., DECAMBOX P., MAUCHIEN P. - Direct and fast determination of uranium in human urines by time-resolved laser-induced spectrofluorometry. Appl. Spectrosc., 1991, 45, 116-118.

[44] MOULIN C., DELORME N., BERTHOUD T., MAUCHIEN P. - Double beam thermal lens spectroscopy for actinides detection and speciation. Radiochim. Acta, 1988, 44/45, 103-106.

[45] MOULIN C., ROUGEAULT S., HAMON D., MAUCHIEN P. - Uranium determination by remote time-resolved laser-induced fluorescence. Appl. Spectrosc., 1993, 47, 2007-2011.

[46] MOULIN C., DECAMBOX P., MAUCHIEN P. - TRLIF for curium trace determination. Radiochim. Acta, 1989, 48, 23-28.

[47] MOULIN V., TITS J., MOULIN C., DECAMBOX P., MAUCHIEN P., DE RUTY O. Complexation behaviour of humic substances toward actinides studied by TRLIF. Radiochim. Acta, 1992, 58/59, 121-128.

[48] MYASOEDOV B.F., NOVIKOV Y.P., MARUNKOV A.G., SINKOV S.I., CHEKALIN N.V. - Use of multiphoton ionization of molecules for the determination of neptunium traces in solution. J. Radioanal. Nucl. Chem., 1988, 121, 337-343.

[49] POLLARD P.M., EWART F.T., LIEZERS M., McMILLAN J.W., THOMASON H.P. - The development of a laser induced photoacoustic facility for actinide speciation. AERE-R-12875, Harwell, 1988.

[50] PRADET B., ROCHE C., REMY B., BERTHOUD T. - Dispositif pour la détermination par fluorescence moléculaire de la concentration d'un composant à surveiller dans un mélange gazeux en circulation. Brevet français 89/133778, 1989.

[51] RADZIEWSKI L.J., CREMERS D.A. - Laser-induced plasmas and applications. New York : M. Dekker, 1989.

[52] REMY B. - Méthode d'étalonnage pour l'analyse en ligne de l'iode et des organoiodés en phase gazeuse par fluorescence à excitation laser. Rapport CEA-R-5467, 1988.

[53] REMY B., FLEUROT B., MAUCHIEN P. - Analyses d'ultratraces par fluorescence induite par laser dans un four de graphite. Rapport technique SPS/LSLA-88, 1990.

[54] REMY B. - Fluorescence atomique excitée par laser en four de graphite : optimisation des paramètres analytiques. Rapport technique SPS/LSLA-77, 1993.

[55] TRAUTMANN N., HERMANN G., RIMKE H., AMES F., MANG M., MUHCLEK C., OTTEN E.W., REKLAU D., RUSTER W., SATTELBERGER P., SCHEERER F. - Low level detection of actinides by laser resonance photoionization. Radiochim. Acta, 44/45, 107-110.

[56] TRAUTMANN N., ALBUS F., AMES F., KLUGE H.J., KRAB S., SCHEERER F., SURI M.B., VENUGOPALAN A., DEIBENBERGER R., KOHLER S., RIEGEL J., URBAN F.J., KIRCHNER R. - A highly efficient and selective laser source by resonance ionization spectroscopy. Inst. Phys. Conf. Ser. 1992, 128(9), 313-316.

[57] PEUSER P., GABELMANN H., LERCH M., SOHNIUS B., TRAUTMANN N., HERMANN G., WEBER M., DENSCHLAG H.O., RUSTER W., BONN J. - Detection methods for trace amounts of plutonium. In : Methods of low-level counting and spectrometry, Berlin 6-10 April 1981. Vienne : AIEA, 1981, 257-262.

[58] TRAUTMANN N., ALBUS F., AMES F., OTTEN E.W., REHKLAU D., SCHEERER F., HERMANN G., RIEGEL J., MUHLECK C., RIMKE H., SATTELBERGER P. - A resonance ionization mass spectrometer as an analytical instrument for trace analysis. Nucl. Instrum. Methods Phys. Res., 1989, A281, 547-558. 
[59] TRAUTMANN N., MONZ L., HOHMANN R., KLUGE H.J., KUNZE S., LANTZSCH J., OTTEN W., PASSLER G., SENNE.P., STENNER J., STRATMANN K., WENDT K., ZIMMER K., HERMANN G., WALTER K. - Colinear resonance ionization spectroscopy for the determination of strontium-90 and strontium-89 in environmental samples. Inst. Phys. Conf., 1992, 128(6), 225-228.

[60] TRAUTMANN N., MONZ L., HOHMANN R., KLUGE H.J., KUNZE S., LANTZSCH J., OTTEN W., PASSLER G., STENNER J., STRATMANN K., ZIMMER K., HERMANN G., WALTER K. - Determination of term energy, hyperfine structure and lifetime of Sr Rydberg levels by resonance ionization spectroscopy in colinear geometry. Inst. Phys. Conf., 1992, 128(2), 87-90.

[61] TRAUTMANN N., MONZ L., HOHMANN R., KLUGE H.J., KUNZE S., LANTZSCH J., OTTEN W., PASSLER G., SENNE P., STENNER J., STRATMANN K., WENDT K., ZIMMER K., HERMANN G., WALTER K. - Fast, low level detection of strontium-90 and strontium-89 in environmental samples by colinear resonance ionization spectroscopy. Spectrochim. Acta (à paraître).

[62] WINEFORDNER J., SIMEONSSON J.B., NG K.C. - Laser-induced fluorescence of rare earth elements in the inductively coupled plasma. Anal. Chim. Acta, 1992, 258, 73-81.

[63] YOUNG J.P., SHAW R.W., SMITH D.H. - Resonance ionization mass spectrometry. Anal. Chem., 1989, 61, 1271-1279.

[64] ZOOK A.C., COLLINS L.H. - Application of a direct method for the determination of trace uranium in safeguards samples by pulsed laser fluorometry. Mikrochim. Acta Part II, 1981, $5 / 6,457-461$. 